\title{
Optical frequency comb generation induced by gain-through-losses modulation instability in passive optical cavities
}

\author{
Florent Bessin ${ }^{1}$, Auro M. Perego ${ }^{2, *}$, Kestutis Staliunas ${ }^{3,4}$, Sergei K. Turitsyn ${ }^{2}$, Alexandre Kudlinski', Matteo \\ Conforti $^{1}$ and Arnaud Mussot ${ }^{1}$ \\ 1. Univ. Lille, CNRS, UMR 8523-PhLAM - Physique des Lasers Atomes et Molécules, F-59000 Lille \\ 2. Aston Institute of Photonic Technologies, Aston University, Birmingham, B4 7ET, UK \\ 3. Institució Catalana de Recerca i Estudis Avançats, Pg. Lluis Companys 23, 08010, Barcelona, Spain \\ 4. Departament de Física i Enginyeria Nuclear Universitat Politécnica de Catalunya, 08222, Terrassa, Spain \\ 5. Novosibirsk State University, Novosibirsk, 630090, Russia \\ *a. perego1@aston.ac.uk
}

Optical frequency combs consist in a set of equally spaced mutually coherent laser frequency lines with a wealth of applications in the most diverse fields of photonics technology including metrology, astrophysics and molecular spectroscopy. Mode-locked lasers and passive driven resonators are the most relevant platforms for their generation. In Kerr resonators, in general frequency combs are generated through four wave mixing triggered by modulation instability (MI) in the anomalous dispersion regime [1]. In this case however the comb repetition rate is not tuneable but it is simply determined by the cavity free spectral range. We present here experimental results about a new method for frequency combs, with tuneable repetition rate, generation in normal dispersion externally driven passive optical resonators with Kerr (cubic) nonlinearity. We exploit the gain-through-losses (GTL) process to excite a modulation instability (MI) which initiates the comb formation. GTL enables energy transfer from a powerful pump field to sidebands frequencies thanks to frequency asymmetric spectral losses for signal and idler waves. The lossy modes themselves are counterintuitively amplified in virtue of the losses presence [2]. We performed an experiment considering a ring fibre resonator of $104 \mathrm{~m}$ length, made with a normal dispersion fiber with group velocity dispersion coefficient $\beta_{2}=0.5 \mathrm{ps}^{2} / \mathrm{km}$. The resonator was pumped at $1545 \mathrm{~nm}$ by a continuous wave laser. A fiber Bragg grating (FBG) with reflection peak located at $400 \mathrm{GHz}$ frequency shift from the pump was used in reflection as a filter with spectral asymmetry with respect to the pump, inducing losses only to the signal (the filter causes no losses to the idler). The GTL MI causes first amplification of frequencies located close to the maximum losses (signal) and to their symmetric with respect to the pump wavelength (idler), which in turn generate higher order harmonics through a cascaded four-wave mixing process hence generating the comb (See Fig. 1(a)). The presence of the unavoidable filter phase contribution is responsible for the shift of the first sideband from the frequency corresponding to the maximum attenuation of the filter. The number of comb lines is limited by the low finesse of our resonator, but could be potentially improved in the future. In the time domain, the waveform corresponds to a train of pulses on the finite background (See Fig. 1(b)). The pulses have a repetition rate related to the inverse of the frequency shift between the first GTL sideband and the pump. Due to the fact that the GTL MI induces amplification of sidebands located close to the filter maximum losses frequency, by changing the detuning between pump and filter it is possible to tune the comb repetition rate. We have indeed verified a tuneability of the comb repetition rate by approximately $100 \mathrm{GHz}$.
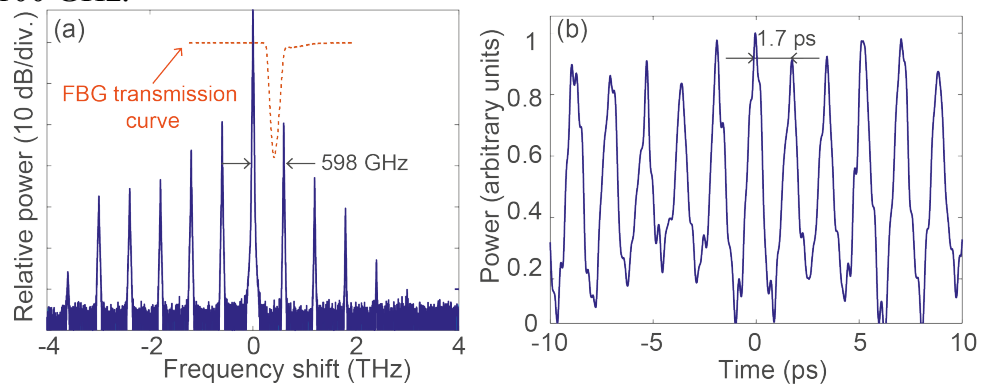

Fig. 1 Experimentally observed frequency comb (a), the temporal trace consists in a train of pulses with a repetition rate given by the inverse of the comb lines spacing (b).

Our method provides a simple passive technique to generate tuneable frequency combs in the normal dispersion regime (avoiding dispersion engineering) and can be extended in the future to monolithic micro resonators too.

\section{References}

[1] T. J. Kippenberg, R. Holzwart and S. Diddams, "Microresonator-based optical frequency combs," Science 332, 555-559 (2011).

[2] A. M. Perego, S. K. Turitsyn and K. Staliunas, "Gain through losses in nonlinear optics," Light: Science \&

Applications 7, 43 (2018). 